\title{
Long-term time-course of nocturnal breathing disorders in heart failure patients
}

\author{
G.D. Pinna*, R. Maestri*, A. Mortara\#, P. Johnson ${ }^{\star}$, D. Andrews ${ }^{\star}$, P. Ponikowski ${ }^{+}$, \\ T. Witkowski ${ }^{+}$, M.T. La Rovere* and P. Sleight
}

ABSTRACT: Some important aspects of clinical manifestations of nocturnal breathing disorders in heart failure (HF) patients are still unknown. We questioned whether the severity of these disorders, first, is stable over time; secondly, shows any systematic trend; and, thirdly, can be predicted over time by a single baseline measurement.

We studied 79 stable, optimally treated, moderate-to-severe HF patients who performed a monthly cardiorespiratory recording during 1-yr follow-up. According to their behaviour over time, nocturnal breathing disorders were classified as persistent, absent or occasional.

During follow-up, clinically relevant breathing disorders were persistent in $\sim 50 \%$ of the patients, absent in $<20 \%$ and occasional in $\sim 30 \%$. Increasing/decreasing trends were rarely observed. The positive and negative predictive value of baseline measurement for persistent behaviour over time ranged, respectively, from $71 \%$ to $91 \%$ and from $91 \%$ to $95 \%$, depending on different levels of severity of breathing disorders.

A large portion of HF patients experience persistent clinically significant nocturnal breathing disorders over long periods of time. Breathing disorders occur irregularly in about one-third of the patients and are negligible in a minority of them. Rarely do they show a steady increase or decrease over time. A single baseline recording predicts a persistent behaviour with moderate-tohigh accuracy.

KEYWORDS: Heart failure, home monitoring for sleep apnoea, sleep-disordered breathing

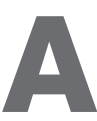
large body of recent evidence indicates that nocturnal breathing disorders are very common in heart failure (HF) patients [1, 2], and are associated with increased mortality independent of major clinical predictors [2-5]. Regardless of their origin (either central or obstructive), these disorders typically manifest as rhythmic rises and falls in tidal volume $(V \mathrm{~T})$, with hyperpnoeic phases followed by apnoeic or hypopnoeic phases [6, 7]. Due to the cyclical fluctuation in ventilation, this pattern has traditionally been referred to as periodic breathing (PB) [8].

Changes in ventilation during PB cause recurrent rises in arterial carbon dioxide levels and falls in oxygen tension, accompanied by cyclic deterioration in cardiac haemodynamics [9]. Intermittent hypercapnia and hypoxia, in turn, are likely to trigger repetitive arousals, with consequent sleep fragmentation [10, 11]. As a result, in patients with HF, nocturnal breathing disorders may further compromise cardiopulmonary function, and increase an already heightened sympathetic drive $[10,12]$.
Although most clinical manifestations of nocturnal breathing disorders in $\mathrm{HF}$ patients are well described, some important aspects await discovery. In this study, we investigated the time-course of breathing disorders over long periods of time (several months), which, to the best of our knowledge, has never been addressed in previous research. In particular, we questioned the following. 1) Are nocturnal breathing disorders stable over time? 2) Do changes over time, if any, show a systematic trend? 3) Can we predict the time behaviour of breathing disorders from a single baseline measurement? The answers to these questions should contribute to a better understanding of the clinical impact of breathing disorders in these patients, and improve the interpretation of their measurement in clinical practice.

\section{METHODS}

\section{Study subjects}

The subjects for this study were a random sample of 174 patients from the original $397 \mathrm{HF}$ patients in whom we had investigated the pathophysiological and clinical relevance of simplified monitoring of nocturnal breathing disorders [5] (as

\section{AFFILIATIONS}

*Dept of Biomedical Engineering and Cardiology, Salvatore Maugeri Foundation-IRCCS, Scientific Institute of Montescano, Montescano (PV), and

\#Dept of Cardiology, Policlinic of Monza, Monza, Italy.

"Nuffield Dept of Medicine, John Radcliffe Hospital, University of Oxford, Oxford, UK.

${ }^{+}$Dept of Cardiology, Clinical Military Hospital, Wroclaw, Poland.

\section{CORRESPONDENCE}

G.D. Pinna

Servizio di Bioingegneria

Fondazione S. Maugeri

IRCCS

Istituto Scientifico di Montescano 27040 Montescano (PV)

Italy

E-mail: giandomenico.pinna@fsm.it

Received:

April 232009

Accepted after revision:

June 032009

First published online:

July 022009 
part of research activities of the European Community multicentre Home or Hospital in Heart Failure $(\mathrm{HHH})$ trial (European Community grant QLGA-CT-2001-02424) [13]). Inclusion criteria were as follows: 1) age 18-85 yrs, 2) New York Heart Association class II-IV, 3) left ventricular ejection fraction $\leqslant 40 \%$, 4) one or more hospital admission for HF or decompensation in the previous 12 months, and 5) abnormal Echo diastolic pattern. Exclusions were as follows: 1) myocardial infarction, cardioverter-defibrillator implantation or revascularisation in the previous 6 months, 2) angina or ischaemia requiring revascularisation, 3) implanted ventricular or atrial pace-maker (except DDD pacemakers with good sinus activity, as we also wished to assess heart rate variability), and 4) severe survival-limiting pathology.

In the HHH study, a subsample of the patients were randomly assigned to perform a 24-h cardiorespiratory recording at home every month [13]. As selected subjects also had a baseline recording, a total of 13 recordings were therefore scheduled for each patient in this arm. Follow-up was at $1 \mathrm{yr}$. A full description of the protocol of the $\mathrm{HHH}$ study can be found in previous reports [13].

\section{Protocol}

Cardiorespiratory recordings were carried out using a simplified device suitable for self-management by the patients at home (Report-24; FM, Monza, Italy), and were transmitted to the analysis centre through a modem connection [14]. A singlelead ECG signal and a respiratory signal (bio-impedance technique) were obtained using three standard ECG electrodes, while a separate sensor detected body position and movement. A validation of this device was carried out in a previous investigation [5].

At enrolment, patients were carefully instructed in the use of telemonitoring devices, and telephone assistance was provided by the study nurse during the follow-up. We have previously shown that $22 \%$ of scheduled recordings at home were not practicable, mainly due to technical problems, logistical difficulties or hospitalisations [14]. Patients carried out $92 \%$ of practicable recordings, thus indicating excellent compliance [14].

All recordings were carefully checked by the signal analyst, and a request for repetition (a maximum of two) was issued to the patient in case of poor signal quality. Night-time was visually identified as the time in bed, based on the body position sensor, between 22:00 hand 08:00 $\mathrm{h}$. The respiratory signal was analysed using dedicated software developed for the study [15], and a set of indexes of breathing disorders were computed. According to the trial protocol, these measurements were available for clinical management only in half of the patients selected at random [13]. In case of severe breathing disorders, treatment by nasal oxygen $\left(3 \mathrm{~L} \cdot \mathrm{min}^{-1}\right)$ or theophylline $\left(3.3 \mathrm{mg} \cdot \mathrm{kg}^{-1} \cdot \mathrm{day}^{-1}\right)$ was suggested. However, the final decision (to change treatment or not) was up to the monitoring physician [13].

Standard clinical and laboratory examinations, including ECG, blood sample determination and echocardiogram, were performed at baseline.

\section{Measurements}

After removal of noise and artefacts, a continuous uncalibrated $V \mathrm{~T}$ signal was derived from the acquired respiratory signal
$[5,15]$. Segments with poor signal quality were discarded. Apnoeas and hypopnoeas were defined as, respectively, a $>90 \%$ and a $>50 \%$ reduction in $V \mathrm{~T}$ lasting $\geqslant 10 \mathrm{~s}$. The apnoea/ hypopnoea index (AHI) was computed as the number of apnoeas and hypopnoeas per hour. PB was defined as a sustained $(\geqslant 3 \mathrm{~min})$ oscillation of $V \mathrm{~T}$ with $>25 \%$ reduction between peak and trough values. This threshold has been successfully used in our previous investigations on the prognostic value of night-time and daytime PB [5, 16]. Finally, we computed the total duration of nocturnal PB. All respiratory events were first detected and estimated by an automatic procedure and then interactively checked and edited by an experienced analyst.

In order to categorise the severity of breathing disorders, the AHI of each patient was dichotomised according to the cut-off points of $\geqslant 5 \cdot h^{-1}$, which is commonly used to detect the presence of clinically significant sleep apnoea [1, 4], and $\geqslant 15 \cdot \mathrm{h}^{-1}$, which is commonly used to detect moderate-to-severe sleep apnoea [1, 2]. Conversely, a PB duration $\geqslant 60 \mathrm{~min}$ was considered to indicate clinically relevant $\mathrm{PB}$, while a $\mathrm{PB}$ duration $\geqslant 120 \mathrm{~min}$ was considered severe PB. The latter cutoff has recently been shown to identify a group of patients at high risk of cardiac events [5].

\section{Criteria for the assessment of the time-course of breathing disorders}

In order to assess the time-course of nocturnal breathing disorders, we defined a set of strict acceptance criteria based on the following: 1) the minimum length of good-quality signal in each recording $(4 \mathrm{~h}), 2)$ the minimum length of follow-up (4 months), and 3 ) the maximum rate of missing measurements (30\% of scheduled recordings). The latter criterion relies on the fact that some recordings could not be carried out due to hospitalisations or technical/logistical problems, while some others, although carried out by the patient, did not satisfy the signal quality criterion. Hence, for instance, patients with 12-, $10-, 8-, 6-$ and 4-month follow-up were included in the study if they had at least nine, eight, six, five and four good quality recordings, respectively.

\section{Statistical analysis}

Comparisons between groups were carried out by the MannWhitney U-test or Chi-squared test if the examined variable was, respectively, continuous or categorical. All tests were two-tailed and a p-value $<0.05$ was considered statistically significant.

Linear regression analysis was performed in each patient to test for the presence of an increasing or decreasing trend in PB duration or AHI during the follow-up.

A given grade of severity of disordered breathing (e.g. AHI $\geqslant 15 \cdot h^{-1}$ ) was defined as "persistent" if it occurred in $>50 \%$ of follow-up recordings, whereas it was defined as "absent" if it did not occur in any follow-up recording. Breathing disorders that were neither persistent nor absent were defined as "occasional". A set of representative examples are given in figs 1 and 2 for PB duration and AHI, respectively.

To assess the predictive value of baseline evaluation of breathing disorders in the prediction of their time behaviour during the follow-up, we analysed the relationship between 



FIGURE 1. Representative examples of the time-course of patterns of nocturnal breathing disorders, as assessed by periodic breathing (PB) duration during 1-yr follow-up of five patients (a-e). _— : valid measurements; ------joins points where measurements were not valid, either because they were missing or the duration of good quality signal was too short (i.e. $<4 \mathrm{~h}$ ), but the previous and following measurements were valid; …..... the two thresholds used in the study to categorise the severity of breathing disorders, namely $60 \mathrm{~min}$ and $120 \mathrm{~min}$. For each grade of severity, the breathing disorder was defined as "persistent" if it occurred in $>50 \%$ of valid follow-up recordings, while it was defined as "absent" if it did not occur in any follow-up recording. Breathing disorders that were neither persistent nor absent were defined as "occasional". a) Persistent PB duration $\geqslant 60 \mathrm{~min}$ (PB 60) and PB duration $\geqslant 120$ min (PB_120); b) absent PB_60 and PB_120; c) occasional PB_60 and PB_120; d) persistent PB_60 and PB_120 with a significant increasing trend $(p=0.006)$; and e) persistent PB 60 and PB 120 with a significant decreasing trend $(p=0.015)$
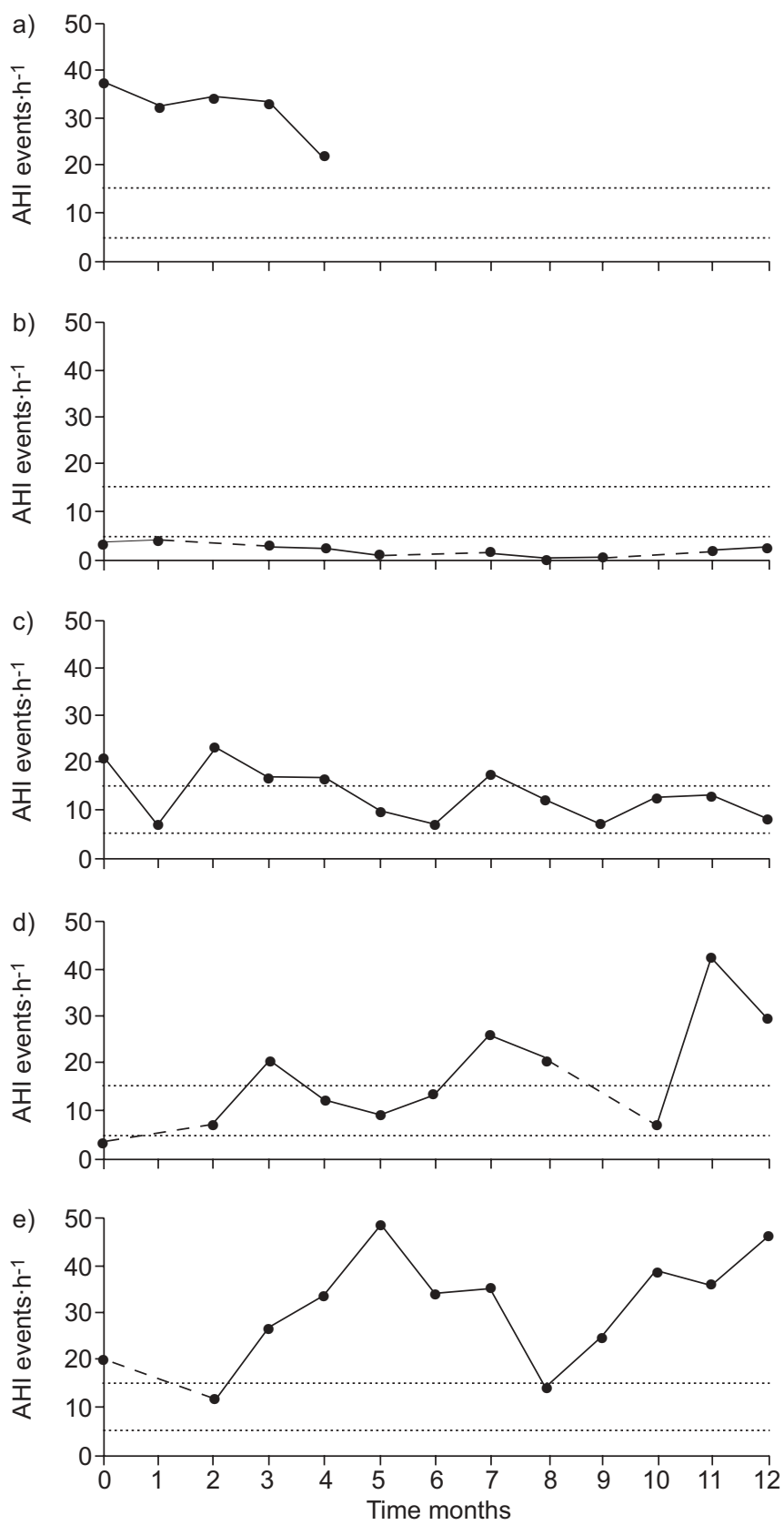

FIGURE 2. Representative examples of the time-course of patterns of nocturnal breathing disorders, as measured by the apnoea/hypopnoea index (AHI) during 1-yr follow-up of five patients (a-e). _—_ valid measurements; -------: joins points where measurements were not valid, either because they were missing or the duration of good quality signal was too short (i.e. $<4 \mathrm{~h}$ ), but the previous and following measurements were valid; ….....: the two thresholds used in the study to categorise the severity of breathing disorders, namely $5 \cdot h^{-1}$ and $15 \cdot h^{-1}$. For each grade of severity, the breathing disorder was defined as "persistent" if it occurred in $>50 \%$ of valid followup recordings, while it was defined as "absent" if it did not occur in any follow-up recording. Breathing disorders that were neither persistent nor absent were defined as "occasional". a) persistent $A H I \geqslant 5 \cdot h^{-1}$ (AHI 5) and $A H I \geqslant 15 \cdot h^{-1}$ (AHI_15). This patient was hospitalised for worsening of heart failure just before the last measurement, and then re-hospitalised twice within the next 40 days before dying of cardiac death. b) Absent AHI 5 and AHI_15; c) persistent AHI_5 and occasional AHI_15; d) persistent AHI_5 and occasional AHI_15 with a significant increasing trend ( $p=0.02)$; and $\mathrm{e})$ persistent AHI 5 and AHI_ 15 with large swings in the severity of abnormal breathing. 
severity of disorders at baseline (i.e. PB duration $\geqslant 120 \mathrm{~min}$ ), and persistence of the same degree of severity during the follow-up. The positive and negative predictive values were computed.

Due to skewness in many variables, descriptive statistics are given as median (lower quartile-upper quartile).

\section{RESULTS}

\section{Included and excluded patients}

$95(55 \%)$ patients out of the 174 enrolled did not fulfil the criteria for the assessment of the time-course of breathing disorders and were therefore excluded from analysis. Baseline demographic, clinical and breathing disorders characteristics of included and excluded patients are reported in table 1 . The two groups had very similar distributions in almost all variables, including therapy. Only a few variables, such as body mass index, heart rate, left ventricular end-diastolic

\begin{tabular}{|c|c|c|c|}
\hline \multirow[t]{2}{*}{ TABLE 1} & \multicolumn{3}{|c|}{$\begin{array}{l}\text { ics of baseline demographic, clinical } \\
\text { ng disorders in patients included and } \\
\text { om the study (because of limited or } \\
\text { data) }\end{array}$} \\
\hline & $\begin{array}{l}\text { Included } \\
\text { patients }\end{array}$ & $\begin{array}{l}\text { Excluded } \\
\text { patients }\end{array}$ & $\mathrm{p}$-value \\
\hline Subjects $n$ & 79 & 95 & \\
\hline Age yrs & $59(53-67)$ & $59(52-68)$ & 0.79 \\
\hline Male \% & 89 & 87 & 0.80 \\
\hline NYHA class $\geqslant$ III \% & 41 & 44 & 0.62 \\
\hline Ischaemic aetiology \% & 48 & 56 & 0.31 \\
\hline $\mathrm{BMI} \mathbf{k g} \cdot \mathrm{m}^{-2}$ & $25.7(23.6-27.5)$ & $27.0(24.1-30.1)$ & 0.04 \\
\hline Heart rate bpm & $70(62-78)$ & $77(70-86)$ & 0.0006 \\
\hline SBP $\mathrm{mmHg}$ & $111(100-120)$ & $116(105-129)$ & 0.26 \\
\hline DBP $\mathrm{mmHg}$ & $70(70-80)$ & $70(70-80)$ & 0.93 \\
\hline LVEF \% & 27 (23-33) & $28(22-35)$ & 0.42 \\
\hline LVEDD mm & $68(62-75)$ & $65(59-71)$ & 0.02 \\
\hline BUN mg $\cdot \mathrm{dL}^{-1}$ & $44(36-52)$ & $41(34-51)$ & 0.41 \\
\hline Sodium $\mathrm{mEq} \cdot \mathrm{L}^{-1}$ & $140(139-143)$ & $140(137-142)$ & $0.01^{\#}$ \\
\hline Creatinine $\mathrm{mg} \cdot \mathrm{dL}^{-1}$ & $1.10(1.01-1.47)$ & $1.14(0.90-1.37)$ & 0.32 \\
\hline Potassium mEq $\cdot \mathrm{L}^{-1}$ & $4.3(4.0-4.5)$ & $4.4(4.0-4.8)$ & 0.08 \\
\hline ACE/ARB \% & 92 & 91 & 0.66 \\
\hline Diuretics $\%$ & 82 & 92 & 0.07 \\
\hline$\beta$-blockers \% & 82 & 84 & 0.73 \\
\hline Digoxin \% & 23 & 31 & 0.25 \\
\hline Anti-aldosterone \% & 51 & 63 & 0.10 \\
\hline PB duration min & $61(25-151)$ & $46(15-112)$ & 0.09 \\
\hline AHI events $\cdot h^{-1}$ & $7.0(2.7-16.3)$ & $5.4(1.7-12.0)$ & 0.15 \\
\hline
\end{tabular}

Data are presented as median (interquartile range), unless otherwise indicated. NYHA: New York Heart Association; BMI: body mass index; SBP: systolic blood pressure; DBP: diastolic blood pressure; LVEF: left ventricular ejection fraction; LVEDD: left ventricular end-diastolic diameter; BUN: blood urea nitrogen; ACE: angiotensin-converting enzyme inhibitors; ARB: angiotensin II receptor blockers; PB: periodic breathing; AHI: apnoea/hypopnoea index. ${ }^{*}$ : this apparent contradiction between the similar median values but a significant $p$-value is simply due to the large number of values at 140 . Indeed, the mean $\pm S D$ in the two groups was $140.6 \pm 3.3$ and $139.2 \pm 3.9$ diameter and sodium, were statistically different but the difference was clinically negligible. None of the included patients received treatment for breathing disorders during the follow-up.

There was a much higher proportion of dropouts in the excluded patients compared with those included (17\% versus $1 \% ; p=0.0006)$, which was indeed a major cause for not meeting entry criteria for this analysis. Both hospitalisations for worsening HF and mortality were very similar in the two groups $(14 \%$ and $17 \%(p=0.60)$ and $9 \%$ and $7 \%(p=0.72)$ for included and excluded patients, respectively).

\section{Recordings in included patients}

The total number of available and good quality recordings in included patients was 765 , i.e. $81 \%$ of those practicable in individual follow-ups. Most subjects (77\%) had nine or more valid recordings, $20 \%$ had between five and eight, and 3\% had only four valid recordings. The total time analysed during the night in valid recordings was $6.3(5.4-7.3) \mathrm{h}$.

\section{Time-course of breathing disorders}

A significant trend to increasing PB duration or AHI during the follow-up was found in nine (11\%) and seven (9\%) patients, respectively. Conversely, a decreasing trend was found in six $(8 \%)$ and three $(4 \%)$ patients, respectively.

The proportion of patients showing persistent, absent or occasional nocturnal breathing disorders according to different levels of severity of $\mathrm{PB}$ duration and $\mathrm{AHI}$ are reported in table 2. It can be seen that, regardless of the type of measurement and level of severity, about one-third of the patients occasionally experienced breathing disorders within the time frame covered by this study, namely $1 \mathrm{yr}$. Clinically relevant breathing disorders (PB duration $\geqslant 60 \mathrm{~min}$ or $\mathrm{AHI}$ $\left.\geqslant 5 \cdot \mathrm{h}^{-1}\right)$ were absent in a minority of subjects $(19 \%$ and $13 \%$, respectively), and were persistent in about half of the patients ( $43 \%$ and $57 \%$, respectively). Conversely, moderate-to-severe

\begin{tabular}{|c|c|c|c|c|}
\hline \multirow[t]{3}{*}{ TABLE 2} & \multicolumn{4}{|c|}{$\begin{array}{l}\text { Proportion of patients with persistent, absent and } \\
\text { occasional breathing disorders during 1-yr } \\
\text { follow-up\# }\end{array}$} \\
\hline & \multicolumn{2}{|c|}{ PB duration } & \multicolumn{2}{|c|}{ AHI } \\
\hline & $\geqslant 60 \mathrm{~min}$ & $\geqslant 120 \mathrm{~min}$ & $\geqslant 5 \cdot h^{-1}$ & $\geqslant 15 \cdot h^{-1}$ \\
\hline Persistent & 43 & 25 & 57 & 20 \\
\hline Absent & 19 & 43 & 13 & 54 \\
\hline Occasional & 38 & 32 & 30 & 26 \\
\hline
\end{tabular}

Data are presented as \%. PB: periodic breathing; AHI: apnoea/hypopnoea


defined as "persistent" during the follow-up if it occurred in $>50 \%$ of valid recordings, while it was defined as "absent" if it did not occur in any recording Breathing disorders that were neither persistent nor absent were defined as "occasional". Only recordings with at least $4 \mathrm{~h}$ of good quality signal were considered. Patients with a follow-up shorter than 4 months or with a number of valid recordings $<70 \%$ of total scheduled recordings were excluded. 
TABLE 3 Descriptive statistics of the proportion of follow-up recordings with breathing disorders exceeding the severity threshold in patients with a persistent breathing disorder

\begin{tabular}{|c|c|c|c|c|}
\hline & $\begin{array}{l}\text { Persistent } \\
\text { PB duration }\end{array}$ & $\begin{array}{l}\text { Persistent } \\
\text { PB duration }\end{array}$ & $\begin{array}{c}\text { Persistent } \\
\text { AHI }\end{array}$ & $\begin{array}{c}\text { Persistent } \\
\text { AHI }\end{array}$ \\
\hline & $\geqslant 60 \mathrm{~min}$ & $\geqslant 120 \mathrm{~min}$ & $\geqslant 5 \cdot h^{-1}$ & $\geqslant 15 \cdot h^{-1}$ \\
\hline $\begin{array}{l}\text { Patients with persistent } \\
\text { breathing disorder } n\end{array}$ & 34 & 20 & 45 & 16 \\
\hline Minimum-maximum \% & $54-100$ & $56-100$ & $55-100$ & $55-100$ \\
\hline
\end{tabular}

PB: periodic breathing; AHI: apnoea/hypopnoea index.

disorders (PB duration $\geqslant 120 \mathrm{~min}$ and $\mathrm{AHI} \geqslant 15 \cdot \mathrm{h}^{-1}$ ) were absent in about half of the patients ( $43 \%$ and $54 \%$, respectively) and were persistent in about one-quarter of them $(25 \%$ and $20 \%$, respectively).

Table 3 gives the descriptive statistics of the actual proportion of follow-up recordings with a positive test in patients with a persistent behaviour (we recall that the definition of persistence has been based on the criterion of having a given level of severity of breathing disorder in $>50 \%$ of follow-up recordings). It can be seen that in most of the patients, a positive test occurred in a proportion of follow-up recordings far exceeding the $50 \%$ threshold.

When interpreting the results given in table 3, if we consider, for instance, the 20 patients with a persistent PB duration $\geqslant 120 \mathrm{~min}$, in half of them, the percentage of analysed followup recordings satisfying this severity criterion was $>80 \%$ and, in three-quarters of them, it was $>67 \%$.

\begin{tabular}{|c|c|c|c|c|}
\hline \multirow[t]{3}{*}{ TABLE 4} & \multicolumn{4}{|c|}{$\begin{array}{l}\text { Positive and negative predictive values of } \\
\text { baseline evaluation of breathing disorders in the } \\
\text { prediction of their persistence during 1-yr follow- } \\
\text { up }\end{array}$} \\
\hline & \multicolumn{2}{|c|}{ PB duration } & \multicolumn{2}{|c|}{ AHI } \\
\hline & $\geqslant 60 \mathrm{~min}$ & $\geqslant 120 \mathrm{~min}$ & $\geqslant 5 \cdot h^{-1}$ & $\geqslant 15 \cdot h^{-1}$ \\
\hline PPV & 75 & 77 & 91 & 71 \\
\hline NPV & 90 & 95 & 91 & 94 \\
\hline
\end{tabular}

Data are presented as percentages. PB: periodic breathing; AHI: apnoea/ hypopnoea index; PPV: positive predictive value; NPV: negative predictive value. When interpreting the results, if, for instance, a patient has baseline PB duration $\geqslant 120 \mathrm{~min}$, then the probability of having a persistent PB duration $\geqslant 120$ min during the follow-up will be $77 \%$. Conversely, if a patient has a baseline PB duration $<120 \mathrm{~min}$, then the probability of never experiencing a PB duration $\geqslant 120$ min during the follow-up or to experience it occasionally (i.e. $<50 \%$ of the time) will be $95 \%$.

\section{Predictive value of baseline evaluation of breathing disorders}

At baseline, $51 \%$ and $28 \%$ of the patients had a PB duration $\geqslant 60 \mathrm{~min}$ and $\geqslant 120 \mathrm{~min}$, respectively, while $58 \%$ and $25 \%$ had an $\mathrm{AHI} \geqslant 5 \cdot \mathrm{h}^{-1}$ and $\geqslant 15 \cdot \mathrm{h}^{-1}$, respectively. Table 4 reports the positive and negative predictive values of baseline evaluation of breathing disorders for the prediction of a persistent behaviour during the 1-yr follow-up. A high percentage of patients (between $71 \%$ and $91 \%$ ) with a positive test at baseline showed a persistent abnormal breathing pattern of the same severity during the following 12 months. An even higher percentage of patients (between $91 \%$ and $95 \%$ ) with a negative baseline test either never experienced an abnormal breathing pattern of the same severity during the following 12 months or experienced it only occasionally.

\section{DISCUSSION}

This study expands the current knowledge on nocturnal breathing disorders in HF patients by providing novel information on their long-term time-course (over $1 \mathrm{yr}$ ) in the home setting. The study results clearly indicate that abnormal breathing patterns during sleep (PB with/without apnoeas) represent a long-term recurrent disorder in most patients, arising either occasionally or persistently, which only rarely shows increasing or decreasing trends. Our study also shows that a persistent behaviour over time can be predicted with moderate-to-high accuracy by a single baseline recording.

\section{Methodological considerations}

In order to provide sound estimates of the time-course of breathing disorder indexes, we applied strict acceptance criteria on the minimum length of good-quality signal in each recording, the minimum length of the observed follow-up and the maximum rate of missing measurements. Although this procedure led to a drastic reduction of the original sample of patients (by 55\%), the selected subsample had very similar clinical characteristics to those of excluded subjects, thus ensuring that we studied a truly representative sample of the considered population.

$19 \%$ of the total scheduled follow-up recordings in all patients were missing, either because they were not carried out by the patients (mostly due to hospitalisations or technical/logistic 
problems) or because signal quality was not adequate. Taking into account that all recordings were self-managed by the patients at home, without any external specialised support (nurse or technician), this rate of missing data is remarkably low.

As a result of the selected criteria, we analysed on average $6.3 \mathrm{~h}$ during the night while the patient was in bed, a result which is in line with those from traditional sleep studies [4].

\section{Time behaviour of breathing disorders}

Using an AHI $\geqslant 5 \cdot h^{-1}$ as the cut-off value for the detection of clinically relevant breathing disorders $[1,4]$, we found that a small minority of HF patients $(13 \%)$ never develop such disorders during $1 \mathrm{yr}$ follow-up. About one-third of the patients experience such disorders occasionally, while in more than half of them they persist over the long term. It thus appears that clinically relevant nocturnal breathing disorders represent an unwelcome night companion for an extraordinarily high proportion of HF patients over the long term. This finding is even more remarkable considering that these patients were receiving high-quality care, as demonstrated by the intensive treatment plan (table 1).

Severe breathing disorders, as defined by a PB duration $\geqslant 120 \mathrm{~min}$, were persistent in $25 \%$ of the patients. We have recently found (in a sample of 397 patients from the same population) that the presence of such a degree of severity increases the 1-yr risk of cardiac death by about three-fold, after adjustment for most important clinical predictors [5]. Subjects at risk were one-quarter of those studied, the same proportion as found in this study. We are therefore led to speculate that the adverse prognostic impact of these disorders is likely to be due to their steady occurrence during night-time over the long term.

A significant increasing or decreasing trend in the severity of breathing disorders during follow-up was observed in a minority of subjects, ranging from $4 \%$ to $11 \%$, with increases being slightly more common. Although this result is certainly affected by the small sample size for linear regression analysis $(\leqslant 13)$, a visual inspection of the behaviour of breathing disorder indices in individual subjects (see, for instance, figs 1 and 2) confirmed that in most instances the severity of breathing disorders is either rather stable over the follow-up period or fluctuates around a substantially constant mean value.

\section{Inferring the time behaviour of breathing disorders}

It is certainly of clinical interest to know, when screening patients for nocturnal breathing disorders, whether the observed degree of severity of the disorder will continue over time, or merely constitute an occasional event. Indeed, although it has been shown that a single night of respiratory monitoring is representative of the current severity of the disorders in these patients $[17,18]$, there are no previous studies investigating its predictive value over the long term. We addressed this question by computing the predictive value of different levels of severity at baseline for a persistent behaviour during the follow-up. We found a moderate-to-high positive predictive value ranging from $71 \%\left(\mathrm{AHI} \geqslant 15 \cdot \mathrm{h}^{-1}\right)$ to $91 \%\left(\mathrm{AHI} \geqslant 5 \cdot \mathrm{h}^{-1}\right)$, thus indicating that a positive test at baseline heralds, in the majority of patients, persistence of the same degree of severity over time. A markedly higher value was found in the negative predictive value, ranging from $91 \%$ $\left(\mathrm{AHI} \geqslant 5 \cdot \mathrm{h}^{-1}\right)$ to $95 \%$ (PB duration $\geqslant 120 \mathrm{~min}$ ), indicating that a negative test at baseline accurately rules out the occurrence of a subsequent persistent behaviour.

\section{Limitations of the study}

An important limitation of this study is that we could not distinguish between central and obstructive events, and therefore could not assess how the two forms of breathing disorders behave over long periods of time. Previous studies, for instance, have shown that a high proportion of patients shift their type of sleep-disordered breathing over a few consecutive nights [18]. Proper discrimination between central and obstructive sleep apnoea requires the recording of chest and abdominal movements, oronasal air flow and oxygen saturation [19], and, with the current state-of-the-art technology, a technician is required to instrument the patient. In our study, this approach would have entailed a total of 944 referrals to the hospital or outpatient clinic, or, equivalently, the same number of home visits by a qualified technician. Hence, the only practicable approach was to use a simplified recording device suitable to be self-managed by the patient at home, and capable of transmitting recorded data to the analysis centre through standard telephone lines [14]. The price we had to pay for using such simple technology was the lack of information about the type of respiratory events. We believe, however, that such limitation does not affect the main finding of the study, namely that abnormal breathing patterns during sleep, regardless of their type, represent a long-term recurrent disorder in most HF patients.

Another limitation of the study is that we could not monitor the cardiopulmonary function of the patients in parallel with monthly respiratory recordings and therefore could not investigate the potential causal link between, for instance, changes in haemodynamic parameters and changes in the severity and pattern of breathing disorders. Such a link is known to exist, especially for central respiratory events [20]. It should be stressed, however, that only a minority of enrolled patients $(\sim 15 \%)$ showed a significant increasing or decreasing trend in the severity of breathing disorders during the observation period, and the rate of hospitalisations for worsening HF was very low (14\%). Hence it is likely that parallel changes in cardiopulmonary function and breathing disorders occurred in a minority of patients.

\section{Conclusion}

In summary, a large portion $(\sim 50 \%)$ of optimally-treated, moderate-to-severe HF patients experience persistent clinically significant nocturnal breathing disorders over long periods of time (several months). Breathing disorders occur occasionally in about one-third of the patients and are consistently negligible or absent in a minority of them $(<20 \%)$. More severe breathing disorders persist in about one-quarter of the subjects. Regardless of their severity, breathing disorders rarely show a steady increase or decrease over time. When a positive test for breathing disorders is found at baseline, there is a moderate-to-high probability of consistently finding the same degree of severity of abnormal breathing during the 
following year. If, on the contrary, a negative test is found, it is very unlikely that persistent breathing disorders would develop over the following year.

\section{SUPPORT STATEMENT}

The Home or Hospital in Heart Failure $(\mathrm{HHH})$ study received a grant from the European Community (Brussels, Belgium; QLGA-CT-200102424).

\section{STATEMENT OF INTEREST}

None declared.

\section{ACKNOWLEDGEMENTS}

We would like to express our deep gratitude to all investigators (physicians, nurses, technicians) who have enthusiastically worked in the $\mathrm{HHH}$ study, contributing in a crucial way to its success.

\section{REFERENCES}

1 Oldenburg O, Lamp B, Faber L, et al. Sleep-disordered breathing in patients with symptomatic heart failure: a contemporary study of prevalence in and characteristics of 700 patients. Eur J Heart Fail 2007; 9: 251-257.

2 Wang H, Parker JD, Newton GE, et al. Influence of obstructive sleep apnea on mortality in patients with heart failure. J Am Coll Cardiol 2007; 49: 1625-1631.

3 Lanfranchi P, Braghiroli A, Bosimini E, et al. Prognostic value of nocturnal Cheyne-Stokes respiration in chronic heart failure patients. Circulation 1999; 99: 1435-1440.

4 Javaheri S, Shukla R, Zeigler H, et al. Central sleep apnea, right ventricular dysfunction, and low diastolic blood pressure are predictors of mortality in systolic heart failure. J Am Coll Cardiol 2007; 49: 2028-2034.

5 Pinna GD, Maestri R, Mortara A, et al. Pathophysiological and clinical relevance of simplified monitoring of nocturnal breathing disorders in heart failure patients. Eur J Heart Fail 2009; 11: 264-272.

6 Tkacova R, Niroumand M, Lorenzi-Filho G, et al. Overnight shift from obstructive to central apneas in patients with heart failure: role of $\mathrm{PCO}_{2}$ and circulatory delay. Circulation 2001; 103: 238-243.
7 Ryan CM, Bradley TD. Periodicity of obstructive sleep apnea in patients with and without heart failure. Chest 2005; 127: 536-542.

8 Cherniack NS. Apnea and periodic breathing during sleep. N Engl J Med 1999; 341: 985-987.

9 Faber J, Lorimer P, Sergysels R. Cyclic hemodynamic and arterial blood gas changes during Cheyne-Stokes breathing. Intensive Care Med, 1990: 208-209.

10 Spaak J, Egri ZJ, Kubo T, et al. Muscle sympathetic nerve activity during wakefulness in heart failure patients with and without sleep apnea. Hypertension 2005; 46: 1327-1332.

11 Trinder J, Merson R, Rosenberg JI, et al. Pathophysiological interactions of ventilation, arousals, and blood pressure oscillations during Cheyne-Stokes respiration in patients with heart failure. Am J Respir Crit Care Med 2000; 162: 808-813.

12 Bradley TD, Hall MJ, Ando S, et al. Hemodynamic effects of simulated obstructive apneas in humans with and without heart failure. Chest 2001; 119: 1827-1835.

13 Mortara A, Pinna GD, Johnson P, et al. Home telemonitoring in heart failure patients: the $\mathrm{HHH}$ study (Home or Hospital in Heart Failure). Eur J Heart Fail 2009; 11: 312-318.

14 Pinna GD, Maestri R, Andrews D, et al. Home telemonitoring of vital signs and cardiorespiratory signals in heart failure patients: system architecture and feasibility of the HHH model. Int J Cardiol 2007; 120: 371-379.

15 Maestri R, Pinna GD, Robbi E, et al. RESP-24: a computer program for the investigation of 24-h breathing abnormalities in heart failure patients. Comput Methods Programs Biomed 2002; 68: 147-159.

16 La Rovere MT, Pinna GD, Maestri R, et al. Clinical relevance of short-term day-time breathing disorders in chronic heart failure patients. Eur J Heart Fail 2007; 9: 949-954.

17 Oldenburg O, Lamp B, Freivogel $\mathrm{K}$, et al. Low night-to-night variability of sleep disordered breathing in patients with stable congestive heart failure. Clin Res Cardiol 2008; 97: 836-842.

18 Vazir A, Hastings PC, Papaioannou I, et al. Variation in severity and type of sleep-disordered breathing throughout 4 nights in patients with heart failure. Respir Med 2008; 102: 831-839.

19 Iber C, Ancoli-Israel S, Chesson A, et al. The AASM Manual for the scoring of sleep and associated events: rules, terminology and technical specifications. 1st Edn. Westchester, American Academy of Sleep Medicine, 2007.

20 Yumino D, Bradley TD. Central sleep apnea and Cheyne-Stokes respiration. Proc Am Thorac Soc 2008; 5: 226-236. 